DIVISION OF THE HUMANITIES AND SOCIAL SCIENCES CALIFORNIA INSTITUTE OF TECHNOLOGY

PASADENA, CALIFORNIA 91125

SOME PROPERTIES OF HARE VOTING WITH STRATEGIC VOTERS

Peter C. Ordeshook and Langche Zeng

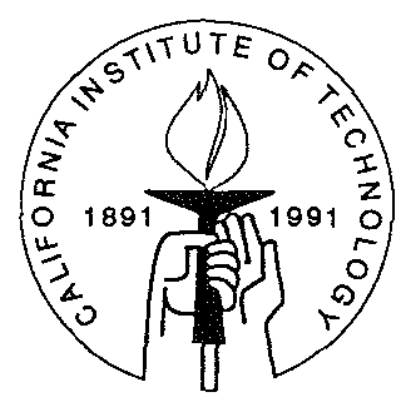

SOCIAL SCIENCE WORKING PAPER 772 


\title{
SOME PROPERTIES OF HARE VOTING WITH STRATEGIC VOTERS
}

Peter C. Ordeshook and Langche Zeng

\begin{abstract}
This essay examines some properties of the Single Transferable Vote (Hare Voting) procedure for electing candidates in multi-member districts under the assumption that all voters are strategic. From the perspective of the most common criterion for evaluating voting procedures - the extent to which they ensure the eventual selection of Condorcet winning candidates - the results we offer in this essay can be interpreted as indictments of STV. Even if we restrict preferences by imposing conditions on attitudes towards risk and assume a strong form of separability, STV is not necessarily incentive compatible and strategic voting does not ensure the selection of Condorcet winning candidates or of Condorcet outcomes. This fact, moreover is not dependent on the existence of "bogus" equilibria - outcomes that exclude Condorcet candidates cannot be avoided under all circumstances even if we limit our analysis to strong or to individually stable equilibria.
\end{abstract}




\section{Some Properties of Hare Voting with Strategic Voters}

\section{Introduction}

American politics are complicated by the necessity, under the winner-take-all format of singlemember districts, for drawing district boundaries to achieve "fair representation" in multi-ethnic and multi-racial polities. The conflicts that arise in this context and the opportunities for lawyers to profitably enter the debate are well known and make it reasonable to search for alternative election procedures. One such alternative is Hare voting or STV (the single transferable vote), which seeks to achieve fairness in the form of proportional representation without necessitating the construction of artificial district boundaries.

Although used on occasion in local contexts, STV has not gained widespread acceptance, in part because it can be difficult to implement in large electorates without computer-aided tabulation technologies and because of the elitist view that electorates are not intellectually equipped to contend with STV's internal mechanics and lengthy ballots. There is, though, another reason for STV's minimal implementation -- the existence of examples in which STV exhibits some undesirable properties. Specifically, Doron and Kronick (1977) show that STV violates monotonicity -- that an increase in first place votes can disadvantage a candidate -- while Brams and Fishburn (1984) offer examples to illustrate that STV need not ensure the selection of a Condorcet winner.

Monotonicity and a guarantee that Condorcet winners are selected are important criteria with which to evaluate any election procedure. Thus, existing research appears to provide a powerful argument against STV's use or for preferring it over other procedures a priori. But the arguments offered by this research are less than compelling since they fail to consider the possibility that voters might act strategically so as to ensure the selection of Condorcet winners. In this essay, then, we explore some of the properties of Hare voting when voters are strategic. We should state at the outset, however, that, owing to STV's strategic complexity, our formal results are limited to some special cases and counter-examples. Nevertheless, our results allow us to formulate informed conjectures about STV's properties.

\section{STV and some Preliminaries}

We begin by introducing some essential notation and with a description of STV in terms of a 5step algorithm. Letting $n$ be the number of ballots (voters in this analysis since we assume that all enfranchised citizens vote), $n_{\mathrm{j}}$ be the number of voters with $t y p e j$ preferences, and $\mathbf{C}=\{a, b, c, \ldots\}$ be the set of candidates, suppose $m<k$ candidates are to be elected or, equivalently, that $k$ candidates compete to fill $m$ seats. Then, following Hoag and Hallett's description (1926), our algorithm is, 
i. $\quad$ Each voter, $j=1,2, \ldots, n$, begins with voting weight $w_{j}=1$;

ii. Each voter $j$ casts a ballot $\mathrm{B}_{\mathrm{j}}$ that ranks the $k$ candidates from first to last;

iii. Letting

$$
W_{i}=\sum_{j=1}^{n} \delta_{j} w_{j}
$$

where $\delta_{\mathrm{j}}=1$ if $j$ 's ballot currently ranks candidate $i$ first, and equals 0 otherwise. Then candidate $i$ is elected if

$$
W_{\mathrm{i}} \geq q=I t\left[\frac{n}{m+1}\right]+1
$$

where $I t$ denotes "the integer portion of."

iv. There are now two possibilities: (1) If $W_{\mathrm{i}}<q$ for all candidates, strike from the ballots the names of those candidates who receive no first place votes. Then delete from the ballot the candidate with the fewest first-place votes. In the event of ties, use a fair lottery to eliminate candidates. (If the number of candidates surviving at any stage equals the number of seats to be filled, then those candidates are elected.) Return to step iii. (2) If $W_{i} \geq q$ for at least one candidate, then elect all such candidates and delete their names from all ballots, and proceed to step $\mathbf{v}$.

v. If candidate $c_{\mathrm{i}}$ is elected, then after deleting $c_{\mathrm{i}}$ from all ballots, set

$$
w_{\mathrm{j}}=\left(W_{\mathrm{i}}-q\right) / H
$$

for all ballots that had ranked $c_{\mathrm{i}}$ first, where $H$ is the number of such ballots. Return to step iii unless all committee positions are filled.

To see the source of our concern over STV's ability to select Condorcet winners if such a winner exists, suppose $k=4, C=\{a, b, c, d\}$ and suppose an electorate of 99 voters is evenly divided across the three preference orders as shown in Example 1.

\begin{tabular}{|lll|}
\hline 33 & 33 & 33 \\
\hline$a$ & $b$ & $c$ \\
$d$ & $d$ & $d$ \\
$b$ & $c$ & $a$ \\
$c$ & $a$ & $b$ \\
\hline Example & 1
\end{tabular}


If $m=3$, then $q=I t[99 / 4]+1=25$ and application of the preceding algorithm to these preferences produces the outcome $\{a, b, c\}$ even though $d$ is the Condorcet winner.

Of course, this example does not establish the superiority of other procedures. If the alternative to STV is three single-member districts, and if each of the above preference orders corresponds to a district, then each district will unanimously elect a candidate other than $d$. Thus, whether an overall Condorcet winner is elected in a single member district system can depend on how district boundaries are drawn.

It is unclear, then, whether the failure to choose the Condorcet candidate is always "a bad thing," since we are hard pressed to identify a "better" outcome than $\{a, b, c\}$. Consider, though, Example 2 in which, if $m=3$, so that $q=25$, the STV outcome is $\{a, b, e\}$ even though $d$ is the Condorcet winner. ${ }^{1}$ In this instance, however, there is no apparent reason to $\operatorname{prefer}\{a, b, e\}$ over $\{a, b, d\}$. Thus, unlike Example 1, Example 2 yields an outcome, $\{a, b, e\}$, that seems dominated normatively by another, $\{a, b, d\}$.

\begin{tabular}{|ccc|}
\hline 38 & 37 & 24 \\
\hline$a$ & $b$ & $c$ \\
$e$ & $d$ & $d$ \\
$d$ & $e$ & $e$ \\
$b$ & $c$ & $a$ \\
$c$ & $a$ & $b$ \\
\hline
\end{tabular}

Example 5

The problem with such examples, though, is the presumption that voting is sincere. Merely applying a procedure to some fixed set of preferences assumes that voters cast ballots consistent with those preferences, whereas Gibbard (1973) and Satterthwaite (1975) establish that if there are three or more outcomes, then there must exist at least one preference profile -- possibly the one under consideration as an example -- such that uniform sincerity is not an equilibrium. The question, then,

1 Note that $a$ and $b$ are immediately elected with 13 and 12 excess votes respectively, which then gives 13 votes to $e, 12$ to $d$, and 24 to $c$. Since no candidate meets the quota, the candidate with the fewest votes, $d$, is eliminated, thereby electing $e$ with 25 first-place votes. 
is whether counter-examples to STV meeting one criterion or another can be sustained as equilibrium outcomes when the electorate is allowed to be strategic.

However, before we can address this matter, we must contend with the fact that we do not yet have a well-defined notion of sincere versus insincere voting since ballots and outcomes are not described in the same way under STV. A ballot, $B_{j}$, is a ranking (complete or partial) of the elements of $\mathbf{C}^{2}$ Thus, if we assume that voters have well-defined preferences over the set of candidates $\mathbf{C}$, we can differentiate between sincere and insincere ballots. But to be correct in our analysis of incentives, we should assume instead that preferences are well-defined over outcomes, which in this instance are sets of elected candidates. That is, if $\mathbf{C}_{\mathrm{m}}$ denotes the set of all subsets of $\mathbf{C}$ of size $m$, then $C \in \mathbf{C}_{\mathrm{m}}$ is an outcome, but knowing a voter's preferences over $\mathbf{C}$ does not necessarily tell us anything about that person's preferences over $\mathbf{C}_{\mathrm{m}}$-- although a voter might prefer $a$ to $b$ to $c$ if forced to choose a single candidate, it is nevertheless possible that this voter prefers the combination $\{b, c\}$ to $\{a, b\}$. Put differently, knowing preferences over $\mathbf{C}_{\mathrm{m}}$ does not tell us which ballots ought to be labeled sincere and which ought to be labeled insincere.

A route out of our problem is to assume that preferences are defined over $\mathbf{C}$-- thereby allowing a "clean" definition of sincere voting -- but to suppose also that there are restrictions on preferences over the elements of $C_{m}$. Specifically, letting $P$ denote strict preference (throughout this essay we simplify matters by assuming that all preferences are strict), consider the following restriction:

R1: If $C-\{c\}=C^{\prime}-\left\{c^{\prime}\right\}$, where $c, c^{\prime} \in \mathbf{C}$ and $C, C^{\prime} \in \mathbf{C}_{\mathrm{m}}$, then $C \mathrm{P} C^{\prime}$ iff $c \mathrm{P} c^{\prime}$.

For example, if $m=2$ and $k=3$, then $\{a, b\} \mathrm{P}\{a, c\} \mathrm{P}\{b, c\}$, if and only if $a \mathrm{P} b \mathrm{P} c$. Notice that if $m$ $=k-1, \mathrm{R} l$ completely constrains the relationship between preferences over $\mathbf{C}_{\mathrm{m}}$ and those over $\mathbf{C}$, whereas if $1<m<k-1$, some latitude remains in this relationship. For example, if $a \mathrm{P} b \mathrm{P} c \mathrm{P} d$, then $\{b, c\} \mathrm{P}\{a, d\}$ and $\{a, d\} \mathrm{P}\{b, c\}$ are both allowed.

If we allow all preference profiles over $\mathbf{C}$, then with or without $\mathrm{R} 1$, all preference profiles over $\mathrm{C}_{\mathrm{m}}$ are admissible and it would appear that Gibbard and Satterthwaite's result applies to establish that insincere voting cannot be precluded $a$ priori. But we have not yet considered a second complicating factor, namely that the set of outcomes also includes lotteries over $\mathbf{C}_{\mathrm{m}}$. If a final outcome corresponds to a tie between two or more candidates and if ties are broken by impartial devices such as coin tosses, then the actual domain of outcomes includes the lotteries over $\mathbf{C}_{\mathrm{m}}$ that such devices allow. Thus, we

2 We assume that all voters cast complete ballots. Brams and Fishburn (1977) show that outcomes may be sensitive to truncation, and this fact ought to be considered in any extension of our analysis. 
ought to ask whether there are any restrictions over preferences for such lotteries in the form of attitudes towards risk that negate the imperatives of any general manipulability result.

The answer to this question is that in general there is no such restriction, but it is nevertheless useful to consider one restriction in particular, because it facilitates the distinction among alternative cases later. That restriction is:

R2: A voter is said to be risk averse if the next-to-last-preferred outcome is preferred to an equi-probable lottery over $\mathrm{C}_{\mathrm{k}-1}$. That is, letting $C_{-\mathrm{i}}=\mathrm{C}-\left\{c_{\mathrm{i}}\right\}$, and letting the subscript $i$ denote a candidate's rank in the voter's preference order, then that voter is said to be risk averse if

$$
\frac{1}{k} \sum_{i=1}^{k} u\left(C_{-i}\right) \leq u\left(C_{-2}\right)
$$

We emphasize that $\mathrm{R} 2$ is severe. For example, if preferences are separable -- if

$$
u\left(C_{-i}\right)=u\left(c_{1}\right)+\ldots+u\left(c_{i-1}\right)+u\left(c_{i+1}\right)+\ldots+u\left(c_{k}\right)
$$

then $\mathrm{R} 2$ requires that

$$
u\left(c_{1}\right) \geq u\left(c_{2}\right)+\sum_{j=3}^{k}\left[u\left(c_{2}\right)-u\left(c_{j}\right)\right]
$$

which requires that the utility difference between a voter's first and second ranked candidates "overwhelms" the combined differences between the second ranked candidate and all remaining candidates.

Throughout the remainder of this essay, we restrict our formal analysis -- but not our examples - to the special case of $m=k-1=2$. We understand that such a restriction does not allow for a general analysis, but this narrow domain is nevertheless sufficient for our purposes -- our results are largely negative and there is no reason to suppose that STV's properties improve as larger values of $k$ and $m$ are considered. That is, if STV has complex and undesirable properties when $m=k-1=$ 2 , we should assume that those properties pervade all STV systems.

\section{Sincerity as a Nash Equilibrium}

We proceed now by noting that the usual analyses of STV take one of two approaches. First, it is shown that applying STV to some fixed set of preferences (ostensibly, sincere preferences) produces 
one type of outcome or another (c.f., Merrill 1984, Doron and Kronick 1977). Alternatively, after postulating preferences and determining the outcome that prevails under sincerity, it is shown how defections from sincerity to insincerity can change the outcome to the benefit of those who defect (c.f. Fishburn and Brams 19xx). Thus, if voters are allowed to be strategic, the first approach yields valid interpretations of STV's potential consequences only if sincerity is a Nash equilibrium. The second approach is valid only if the defections considered are the only ones that will be made by strategic voters -- only if the new assumed strategies are an equilibrium. What we propose, then, is to explore the circumstances under which these implicit assumptions are valid.

We turn first to the issue of determining the circumstances under which sincerity is a Nash equilibrium. Let $V_{\mathrm{i}}$ denote the number of voters who most prefer candidate $i$, let $V_{\mathrm{i}}{ }^{\text {jh }}$ denote the number of voters who prefer $i$ to $j$ to $h$, and let $W_{\mathrm{i}}$ be defined as before. Then Result 1 (which we prove in the appendix), establishes that while there can be circumstances under which sincerity is a Nash equilibrium, there are also circumstances in even a very limited context under which it cannot be an equilibrium:

Result 1: If $m=k-1=2$ and

1. if $R 2$ is satisfied, then a voter who prefers $i P j P h$ will defect from the strategy $n-$ tuple "everyone votes sincerely" if and only if $V_{\mathrm{i}}>q$, and $V_{\mathrm{j}}, V_{\mathrm{h}}<q$ for the remaining two candidates, with

$$
W_{\mathrm{j}}-W_{\mathrm{h}}+\frac{W_{\mathrm{i}}^{\mathrm{jh}}-W_{\mathrm{i}}^{\mathrm{hj}}}{W_{\mathrm{i}}}\left[W_{\mathrm{i}}-q\right] \epsilon[\delta, 1]
$$

where $\delta \geq-1$ depends on i's excess votes and the portion of these votes transferred to $j$;

2. if $R 2$ is not satisfied, the set of preferences that do not allow sincerity to be a Nash equilibrium expands to include those situations in which $V_{\mathrm{i}}=q$ and $V_{\mathrm{j}}, V_{\mathrm{h}}<q$.

One unsurprising implication of Result 1 is that there exists ordinal preferences such that "all voters vote sincerely" is not a Nash equilibrium. In Example 3, with $m=2$ and $q=34$, if any voter who most prefers $a$ to $b$ to $c$ defects unilaterally from sincerity, the outcome is $\{a, b\}$ rather than a lottery between $\{a, b\}$ and $\{a, c\}$. 


\begin{tabular}{|rrrr|}
\hline 18 & 18 & 32 & 32 \\
\hline$a$ & $a$ & $c$ & $b$ \\
$b$ & $c$ & $a$ & $c$ \\
$c$ & $b$ & $b$ & $a$ \\
\hline
\end{tabular}

Example 3

On the other hand, Result $\mathrm{I}$ also tells us that there is a wide range of circumstances under which "all voters vote sincerely" is a Nash equilibrium and it suggests where we might look for such equilibria in situations other than $k=3$. For instance, in Example 1, because all candidates but $d$ exceed the quota, no individual has any incentive to defect from sincerity. Thus, the election of $\{a, b, c\}$ is an equilibrium. To see, moreover, that the existence of sincere equilibria does not depend on the inability of individual voters to be pivotal, suppose we try to elect $d$ in Example 1 by having 8 voters from each of the three preference types reverse the order of their first and second-ranked candidates on their ballots. This switch produces Example 4. Clearly, candidates $a, b$ and $c$ continue to be elected under this ballot profile. Now consider whether a voter who holds the first preference prefers to cast an insincere ballot. If this voter switches the position of $a$ and $d, d$ is elected, but $a$ is eliminated, thereby producing the outcome $\{b, c, d\}$-- the voter's least preferred outcome -whereas moving $b$ and $c$ to the top position produces the same outcome. Thus, none of the twenty four voters who switched from sincere to insincere ballots by ranking $d$ first can gain, and indeed, there are circumstances under which they are hurt by doing so. Moreover, the insincere ballots of these twenty four voters, although they render all other voters pivotal, do not create a situation in which any of these other voters prefers to be insincere. Thus, voting sincerely is an equilibrium even though the Condorcet winner is not elected.

\begin{tabular}{|llllll|}
\hline 25 & 25 & 25 & 8 & 8 & 8 \\
\hline$a$ & $b$ & $c$ & $d$ & $d$ & $d$ \\
$d$ & $d$ & $d$ & $a$ & $b$ & $c$ \\
$b$ & $c$ & $a$ & $b$ & $c$ & $a$ \\
$c$ & $a$ & $b$ & $c$ & $a$ & $b$ \\
\hline
\end{tabular}

Example 4 
In summary, then: Sincere voting can be a Nash equilibrium in STV systems even if the equilibrium outcome fails to elect a Condorcet winning candidate; and this fact does not depend on examples in which no voter is pivotal or in which voters fail to eliminate (weakly) dominated strategies.

\section{Insincerity and Condorcet Winners}

As our summary statement indicates, we are not interested in sincere versus insincere voting for its own sake. Rather, our interest derives from the desire to gain some sense of the circumstances under which STV systems are likely to elect Condorcet winners in the event that such winners exist. But Result 1 also leads us to conjecture that as the number of candidates increases beyond 3, the incentives for insincerity will expand. Even if strong assumptions such as R2 are satisfied, as $k$ grows, the opportunities for making or breaking ties among those candidates not elected on the first ballot will increase, and it is precisely these opportunities that induce voters to cast insincere ballots. This fact, then, raises a new question. Specifically, if voters find it in their interest to vote insincerely, will such voting ensure the election of Condorcet winning candidates?

Unfortunately, Example 5 reveals that the answer to this question is no -- indeed, strategic voting can lead away from the selection of a Condorcet winner.

\begin{tabular}{|llll|}
\hline 18 & 17 & 32 & 32 \\
\hline$a$ & $a(c)$ & $c$ & $b$ \\
$b$ & $c(a)$ & $b$ & $a$ \\
$c$ & $b(b)$ & $a$ & $c$ \\
\hline
\end{tabular}

Example 5

Ignoring the preferences in parentheses, if everyone votes sincerely, $a$ is elected because its vote, 35 , exceeds the quota of 34 -- at which point $b$ is elected because its share of $a$ 's excess vote exceeds $c$ 's share, thereby breaking the tie between $c$ and $b$ in favor of $b$. So the Condorcet winner, $b$, is elected in a sincere ballot. Now, however, consider the incentives to be strategic. Some simple algebra establishes that expression (1) in Result 1 is satisfied only for voters who prefer $a$ to $c$ to $b$, so only they have an incentive to defect from sincere voting. In particular, if any such voter casts a ballot that ranks $c$ first, $a$ is elected again but has no excess votes to transfer to $b$, in which case $b$ is eliminated and $c$ is elected. Moreover, if R2 is satisfied, Result 1 tells us that this new ballot profile with a single insincere voter is an equilibrium. 


\section{Strong Equilibria and Condorcet Winners}

Thus far we have seen that sincere voting can be an equilibrium that fails to elect Condorcet winners, and that insincere voting need not elect such winners, even in equilibrium. What we have not considered, though, is the possibility that other variations of the definition of equilibrium can cause us to modify our conclusions about STV's empirical properties.

To see the problem more clearly, consider Example 6a in which, if $m=1$ (so that $q=51$ ) and if everyone is sincere, the outcome $\{b\}$ prevails even though $c$ is the Condorcet winner, whereas if we allow voters to be strategic, $c$ is elected: ${ }^{3}$ Now. consider Example $6 \mathrm{~b}$, which adds candidates to Example $6 \mathrm{a}$ so that sincere voting continues to select $b$ but unilateral adjustments in rankings and deletions of dominated strategies do not lead to the selection of the Condorcet winner, $c$. Asserting that $b$ is the inevitable final outcome, though, is problematical. First, notice that if all voters of the first type rank $e$ above $a$, then $e$ is elected. But now if all voters of the second type respond by ranking $c$ first, then $c$ is elected. Of course, this reasoning is reminiscent of the "he-thinks" regress that notions of non-cooperative equilibria are designed to resolve. The particular difficulty here, though, is that no individual voter is decisive for the outcome and, therefore, there is no guarantee that that reasoning will proceed in such a way as to lead to one type of equilibrium rather than another. In other words, we may become "trapped" in one type of equilibrium merely because no single voter can have any influence on the outcome.

\begin{tabular}{|rrr|}
\hline 40 & 40 & 20 \\
\hline$a$ & $b$ & $c$ \\
$c$ & $c$ & $b$ \\
$b$ & $a$ & $a$ \\
\hline
\end{tabular}

Example 6a

\begin{tabular}{|lll|}
\hline 40 & 40 & 20 \\
\hline$a$ & $b$ & $d$ \\
$e$ & $c$ & $c$ \\
$c$ & $a$ & $e$ \\
$b$ & $d$ & $b$ \\
$d$ & $e$ & $a$ \\
\hline
\end{tabular}

Example 6b

3 Because it is never advantageous to rank one's least preferred candidate first or second, voters of the first type know that even with strategic voting, candidate $a$ can never be elected. Thus, each such voter should rank $c$ first so as to elect $c$ rather than $b$. That is, all " $a$-voters" insincerely ranking $c$ first on their ballots, and everyone else voting sincerely" is an equilibrium. 
Such examples, then, illustrate a general problem with STV. On the one hand, a great many equilibria are supported by the fact that no individual voter has a positive incentive to alter its strategy. On the other hand, these equilibria cannot be eliminated using refinements like trembling hand perfection or elimination of weakly dominated strategies, because there is always at least one circumstance in which the strategy in question is strictly preferred to all others.

We suspect, nevertheless, that such equilibria are unreasonable predictions or that they provide something less than a firm basis for evaluating election procedures. First, although each voter knows that he or she is unlikely to be pivotal, each voter also knows that its vote is irrelevant unless it is pivotal. Thus, one alternative is to restrict ourselves to examining equilibria in which at least one voter is pivotal. We call such equilibria individually stable.

Second, we know that considerable effort is directed in multi-candidate election campaigns at coordinating the actions of voters. Labor unions, newspapers, and the candidates attempt to educate voters about strategic complexities, especially when it is in their interest to do so, by telling them, for instance, how not to "waste their vote" or how voting for one candidate merely works to the advantage of some undesirable alternative. Thus, it seems reasonable to pay special heed to those equilibria that cannot be upset even if voters can successfully coordinate their actions. There are several ways to approach this second consideration. Myerson (19xx), for instance, introduces the notion of proper equilibria to handle precisely this type of problem; but STV's complexity leaves us perplexed as to how to apply this idea. Hence, we turn instead to Aumann's (1957) notion of a strong equilibrium - a Nash equilibrium that is stable against the coordinated defections of any set of voters.

To see the problems we encounter now with ensuring the selection of a Condorcet winning candidate, consider the following fact:

Remark 1: Even if $\mathbf{C}$ contains a Condorcet winning candidate and even if preferences satisfy $R 1$, it is not necessarily the case that $\mathrm{C}_{\mathrm{m}}$ contains a Condorcet winning outcome.

Suppose individual preferences establish $d$ as the Condorcet winner, but suppose that they also generate a cycle among candidates $a, b$, and $c$. Then if $m=2$, the outcomes $\{a, d\},\{b, d\}$, and $\{c, d\}$ form a top-cycle in $\mathbf{C}_{\mathrm{m}}$, whereas if $m=3$, then $\{d, a, b\},\{d, a, c\}$, and $\{d, b, c\}$ cycle. This is not to say, though, that we cannot induce Condorcet outcomes with appropriate preferences over candidates. For example,

Remark 2: If preferences over $\mathbf{C}$ are single-peaked, then under $R 1, \mathbf{C}_{\mathrm{m}}$ contains a Condorcet winning outcome. 
If preferences over $\mathbf{C}$ are single peaked, then preferences over $\mathbf{C}$ are transitive under simple majority rule. If this transitive order is, say, $1 \mathrm{P} 2 \mathrm{P} 3$, and so on, then the Condorcet outcome in $\mathrm{C}_{\mathrm{m}}$ is $\{1,2, \ldots, m\}$. $^{4}$

The question, now, is whether the notions of individually stable and strong equilibria can rationalize the selection of a Condorcet winning outcome when such an outcome exists. The following two preliminary results, which we state without proof owing to their limited applicability, suggest that the answer to this question is yes:

Result 2: If $m=1$, then in any strong or individually stable equilibrium, the Condorcet winner is elected if such a winner exists.

Result 3: If $m=2$ and $k=3$, if $n_{\mathrm{i}} \geq q$ for at most one candidate, if $R 2$ is not satisfied, then sincere voting is an individually stable or a strong equilibrium only if a Condorcet winner. whenever it exists, is either elected with certainty or with some non-zero probability.

Unfortunately, a single counter-example, Example 7, dashes all hope of extending these results:

\begin{tabular}{|lllll|}
\hline 34 & 32 & 11 & 11 & 10 \\
\hline$b$ & $c$ & $d$ & $d$ & $d$ \\
$a$ & $a$ & $a$ & $c$ & $b$ \\
$c$ & $b$ & $c$ & $a$ & $a$ \\
$d$ & $d$ & $b$ & $b$ & $c$ \\
\hline
\end{tabular}

Example 7

Given these preferences, candidate $a$ is the Condorcet winner and, for $m=2,\{a, c\}$ is a Condorcet outcome - $a$ defeats $b, c$ and $d$; and $c$ defeats both $b$ and $d$. However, sincere voting yields the

4 Notice, however, that $\mathrm{Rl}$ is not sufficiently restrictive to allow us to assert that preferences over $\mathbf{C}_{\mathrm{m}}$ are transitive even if preferences over $\mathbf{C}$ are single peaked. For example, if $\mathbf{C}=\{1,2, \ldots, 12\}$ and $m=4$, then we cannot infer anything about the social preference relation among the outcomes $\{1,2,11,12\},\{3,4,9,10\}$, and $\{5,6,7,8\}$. 
outcome $\{b, c\}--$ candidate $b$ is elected, after which $a$ is eliminated because it has only $b$ 's one excess vote; this vote is then given to $c$ and $c$ is elected.

Our interest in this example, though, derives from the fact that sincere voting is the unique strong equilibrium. To see this, notice that there are only three alternative outcomes, $\{a, c\},\{a, b\}$, and $\{b, d\}$, and we can consider whether any of these outcomes can be realized by coordinated action.

$\{a, c\}$ : Preference types 2,3 , and 4 , with a total of 54 votes, prefer $\{a, c\}$ to $\{b, c\}$. None of these voters, though, can abort the selection of $b$, and thus they can merely substitute the outcome $\{a, b\}$ for $\{b, c\}$.

$\{a, b\}$ : Preference types 1,3 , and 5 , with 55 votes, $\operatorname{prefer}\{a, b\}$ to $\{b, c\}$. Type 1 voters have no incentive to change, even if they coordinate somehow with types 3 and 5 , since any change merely elects $a$ rather than $b$ on the first round. If types 3 and 5 rank $a$ first, so that it is not eliminated after $b$ is elected, $c$ gets 32 votes, $a 23$, and $d 11$, at which point $d$ is eliminated and $c$ is elected (having gained 11 votes from the type 4 voters, who have no incentive to coordinate so as to elect candidate $b$ over $c$ ).

$\{b, d\}$ : Only voters with type 3,4 and 5 preferences prefer $\{b, d\}$ to $\{b, c\}$, but since they are already ranking $d$ first and are not instrumental in the eventual selection of $c$ (recall that $c$ is elected by the type 1 voters after $a$ is eliminated from consideration), they cannot together change the outcome.

Thus, no subset of voters has any incentive or means to coordinate so as bring about an outcome other than $\{b, c\}--\{b, c\}$ corresponds to the unique strong equilibrium.

\section{Conclusion}

From the perspective of some common criteria for evaluating voting procedures, the results we offer in this essay will almost certainly be interpreted as indictments of STV. Even if we restrict preferences with conditions R1 and R2, STV is not necessarily incentive compatible. Moreover, strategic voting does not ensure the selection of Condorcet winning candidates or of Condorcet outcomes. This fact, moreover is not dependent on the existence of "bogus" equilibria -- outcomes that exclude Condorcet candidates cannot be avoided under all circumstances even if we limit our analysis to strong or to individually stable equilibria.

It is not the case, though, that the Condorcet criterion is the unique or even the most important criterion with which to evaluate election procedures. For example, we have not examined the extent to which STV ensures proportionality and we have not compared STV to other procedures. Despite 
the apparent deficiencies described in this essay, STV may yet prove to be a viable alternative to systems that seek to ensure proportionality by other means.

\section{Appendix: Proof of Result 1}

The proof of Result 1 makes use of three lemmas. Our first lemma, though, can be stated without proof, because it concerns the situation in which Hare voting corresponds to a simple 2-candidate contest.

Lemma 1: If $m=1$ and $k=2$, then voting sincerely is (weakly) dominant. Second,

Lemma 2: If $W_{\mathrm{i}}<q$ for all $i$, then $W_{1}=W_{2}=\ldots=W_{\mathrm{n}}=q-1 .^{5}$

Finally,

Lemma 3: If $W_{\mathrm{i}}=q$ for only one $i$ and $W_{\mathrm{j}}<q$ for all $j \neq i$, then either $W_{\mathrm{h}}=q-2$ for one $h \neq i$ and $W_{\mathrm{j}}=q-1$ for all $j \neq i, h$ or $W_{\mathrm{j}}=q-1$ for all $j \neq i .^{6}$

Turning to the proof of Result 1 , suppose $B=\left\{B_{1}, B_{2}, \ldots, B_{n}\right\}$ is any configuration of sincere ballots, and consider any voter, $j$. What we want to establish is the incentives for this voter to deviate from sincerity. Let $C=\{a, b, c\}$, and to simplify matters, assuming, without loss of generality, that this voter holds the preference $a \mathrm{P} b \mathrm{P} c$. We have four cases:

5 If the preconditions of the lemma hold, then $W_{\mathrm{i}} \leq q-1$ for all $i$. If for any $i, W_{\mathrm{i}}<q$, summing up votes across the candidates gives $\Sigma W_{\mathrm{i}}<k q-k$. However, despite the truncation required by It in the definition of $q$, we must have $k q-3 \leq \Sigma W_{\mathrm{i}}$, and these two inequalities cannot be satisfied simultaneously. So equality must hold originally -- that is, $W_{\mathrm{i}}=q-1$ for all $i$, which is a $k$-way tie.

6 Suppose to the contrary that $W_{\mathrm{h}}<q-2$ or $W_{\mathrm{h}} \leq q-2$ for more than one $h$. Then

$$
\begin{aligned}
& \sum_{i=1}^{k} W_{\mathrm{i}} \leq q+(q-3)+(k-2)(q-1) \\
& =k q-k-1=k[n / k]-1<n
\end{aligned}
$$

which is impossible. 
Case 1: $W_{\mathrm{i}}<q$ for all candidates. From Lemma 2, the outcome is a 3-way tie, in which case sincere voting yields an equi-probable lottery over $\{a, b\},\{a, c\}$, and $\{\mathrm{b}, \mathrm{c}\}$. If the voter insincerely votes $b \mathrm{P}$ $a \mathrm{P} c$, candidate $b$ is elected with certainty but has no excess votes to transfer to $a$. Candidate $a$ now has one fewer first-place votes than $c$, so the switch causes $\{b, c\}$ to prevail -- the voter's least preferred outcome.

Case 2: $W_{i}>q$ for one candidate. In this instance there are two subcases:

i. $\quad W_{\mathbf{a}}>q$ : The voter cannot affect the election of $a$. However, if it votes sincerely, $b$ and $c$ 's respective voting weights subsequently are

$$
\begin{aligned}
& W_{\mathrm{b}}+W_{\mathrm{a}}^{\mathrm{bc}} \frac{\left(W_{\mathrm{a}}-q\right)}{W_{\mathrm{a}}} \\
& W_{\mathrm{c}}+W_{\mathrm{a}}^{\mathrm{cb}} \frac{\left(W_{\mathrm{a}}-q\right)}{W_{\mathrm{a}}}
\end{aligned}
$$

Clearly, the voter in question can be decisive for $b$ versus $c$ only if the difference in these weights is less than or equal to 0 but not so small as to preclude the voter from at least creating a tie between $b$ and $c$. By voting insincerely, the voter increases $b$ 's vote by 1 , less the decreased transfer of $a$ 's excess. Candidate $b$ 's net gain, then, is

$$
1-W_{\mathrm{a}}^{\mathrm{bc}}\left[1-\frac{q}{W_{\mathrm{a}}}\right]+\left(W_{\mathrm{a}}^{\mathrm{bc}}-1\right)\left[1-\frac{q}{W_{\mathrm{a}}-1}\right]
$$

So

$$
\delta=\frac{q}{W_{a}-1}-q\left[\frac{W_{a}^{d x}}{W_{a}-1}-\frac{W_{a}^{d x}}{W_{a}}\right]
$$

Notice now that if $n$ is sufficiently large so that $q /\left(W_{\mathrm{a}}-1\right)$ is approximately equal to $q / W_{\mathrm{a}}$, then

$$
\delta \approx-\frac{q}{W_{a}}
$$

so if the value of expression (1) falls in the interval $[\delta, 0]$, then the voter votes insincerely. 
ii. $\quad W_{\mathrm{b}}\left(W_{\mathrm{c}}\right)>q$ : The voter cannot affect the election of $b(c)$, and it cannot improve $a$ 's chances by any insincere ballot.

Case 3: $W_{\mathrm{i}}=q$ for only one candidate. There are three subcases:

i. $\quad W_{\mathrm{a}}=q$. From Lemmas 3 , either $W_{\mathrm{b}}=W_{\mathrm{c}}=q-1$, in which case a sincere ballot yields an equi-probable lottery between $\{a, b\}$ and $\{a, c\}$ and an insincere one yields a lottery between $\{a, b\}$ and $\{b, c\}--$ and the voter prefer the first lottery to the second. Alternatively; if, from Lemma 3, $W_{\mathrm{c}}=q-1$ and $W_{\mathrm{b}}=q-2$, then ranking $b$ first yields an equi-probable lottery over all three possible outcomes. And by R2, the voter prefer the certainty of $\{a, c\}$ to this lottery.

ii. $\quad W_{\mathrm{b}}=q$. Switching to bac merely gives $b$ one more vote and makes the eventual selection of $a$ less likely.

iii. $\quad W_{\mathrm{c}}=q$. Switching to $b a c$ can at best elect $\{b, c\}$, whereas voting sincerely can yield $\{a, c\}$.

Case 4: $W_{\mathrm{i}} \geq q$ for two candidates. We should assume that equality holds for at least one candidate since if inequality holds no individual voter can influence the outcome and voter $j$ in particular has no incentive to deviate from a sincere strategy. So we have three subcases:

i. The two candidates are $a$ and $b$, in which case the voter has no incentive to change its vote since $\{a, b\}$ is the voter's most preferred outcome.

ii. The two candidates are $a$ and $c$. If $W_{\mathrm{a}}=q$, then switching to a ballot that orders the candidates bac can only elect $b$ along with $c$-- the voter's least preferred outcome. If $W_{\mathrm{c}}=q$ and $W_{\mathrm{a}}>q$, then $W_{\mathrm{b}}<q-1$ and switching to bac cannot change the outcome.

iii. The two candidates are $b$ and $c$. A sincere ballot cannot elect $a$, so neither can an insincere one. 


\section{References}

Aumann, Robert J. 1959. "Acceptable Points in Cooperative n-Person Games," in H.W. Kuhn and R.D. Luce, eds., Contributions to the Theory of Games IV, Princeton: Princeton Univ. Press.

Brams, Steven J. and Peter C. Fishburn. 1984 "Some Logical Defects of the Single Transferable Vote," in A.rend Lijphart and Bernard Grofman, eds. Choosing an Electoral System, N.Y.: Praeger.

Doran, Gideon and Richard Kronick. 1977. "Single Transferable Vote: An Example of a Perverse Social Choice Function," American Journal of Political Science, 21,2:303-11

Gibbard, Allen. 1973. "Manipulation of Voting Schemes," Econometrica, 41:587-601.

Hoag, Clarence G and George H. Hallett. 1926. Proportional Representation, N.Y.: Macmillan.

Merrill, Samuel. 1984. "A Comparison of Efficiency of Multicandidate Electoral Systems, American Journal of Political Science, 28,1:23-48.

Myerson, Roger B. 1978. "Refinements of the Nash Equilibrium Concept," Journal of Game Theory, 7,2:73-80.

Satterthwaite, Mark. 1975. "Strategy Proof ness and Arrow's Conditions," Journal of Economic Theory, 187-217. 